\title{
Thinking of the Red Stoneware Art of Jiang Rong
}

\author{
Jianlin $\mathrm{Ni}$ \\ School of Fine Arts \\ Nanjing Normal University \\ Nanjing, Jiangsu, China
}

\begin{abstract}
The theory of "Picturesque" has developed to be a new theory by aestheticians, used to balance the two classic aesthetic categories of "beauty" and "sublime". As the same, the three-dimensional works of typification, which were created by artists with refinement and emotional engagement, would also have characteristics beyond true nature, this is the theory of "Sculpturesque". The most remarkable accomplishment of Jiang Rong`s Red Stoneware Art is bionic art-design and moulding, and the main feature of that is merging the natural shape into some specific functional parts. The artistic style of Jiang Rong is full of fresh, native, vivid, brilliant, and expressed within an inch of our lives; Moreover, the women's fine sentiments emitted by her works is unique, beyond the shapes and colors, Jiang Rong`s art give us the psychological experience of similarity in spirit. Typification is a critical step in representing the natural objects truly. In view of the key fact of expressing is the manipulation towards the emotional engagement of the object, so it may not be able to build the spiritual similarity works upon appearance imitating merely. Jiang Rong`s works make us fully experience that the close relationships between Art and Craft: when the artist owns distinguished artistic taste as well as extraordinary vision, he would use the craft to express his ideas perfectly. From this level, Art is actually a special Craft.
\end{abstract}

Keywords-Jiang Rong; Red Stoneware; HuaHuo; bionic and imitating vessels; Sculpturesque

\section{INTRODUCTION}

In the history of modern aesthetic, there is a special theory known as "picturesque", it originally meant a some beautiful, natural scene that would conjure images of pictures painted by artists, and these would be known as "picturesque scenes", or "photosque beauty". It was developed to be a new theory by aestheticians, and later, used to balance the two classic aesthetic categories of "beauty" and "sublime". The reason why "picturesque" theory was formed was that the nature in works which were created by artists, known as "typification", came from their ability to refine and emotionally engage the subject. It was therefore difficult to find such "typification" of pure beauty from true nature. In that way, the artistic reality of typification could surpass the true natural reality, and the theory could be applied to three-dimensional sculptures. That is to say, the three-dimensional works of typification, which were created by artists with refinement and emotional engagement, would also have characteristics beyond true nature. Therefore when such perfect objects were found in true nature, they were known as "sculpturesque". My understanding of the concept of "sculpturesque" turly manifested itself when I observed the Red Stoneware Art of Jiang Rong.

Jiang Rong (1919-2008) is a famous Chinese contemporary artist who is well known for her YiXing Red Stoneware Art, she has enjoyed a high reputations and status in the Red Stoneware Art field for quite some time. We do not need to use any words to praise her achievement, because those are nothing more than just icing on the cake. In view of her outstanding achievement in not only Chinese art, but also art in general, it is necessary to discuss and compare Jiang Rong`s red stoneware art from a standpoint of Art and History. In this way, we are not only able to objectively restore the artist's achievement, but also to reveal the position of YiXing Red Stoneware Art among the whole field of Art.

In spite of the fact that history can not be changed, there still have some man-made factors which influenced its spreading. An example that Chinese traditional Arts and Crafts has been developed to a very ingenious level early in the time of ancients, but have never been as prized as paintings. What's a worse is that Chinese craftsman were always seen as underprivileged, so that their works were ignored for a long time. The phenomenon is not due to the essence of Art but some cultural factors which are man-made. This may be the reason why some believe that "history is subjective". Therefore, positioning, judging and selecting historical facts are closely associated with historians `vision and qualities. Sufficient commentary and researchful discussion will contribute to understand artists and their works more appropriately.

The most remarkable accomplishment of Jiang Rong`s Red Stoneware Art is bionic art-design and moulding, which is called "HuaHuo" in the industry; besides, she is also good at works imitating various fruits and vegetables for appreciating purpose. As far as the creative philosophy and craftsmanship, the two are exactly the same; so we are able to focus Jiang Rong`s Red Stoneware Art achievement on bionic art-design and imitating creation.

\section{THE THREE-DIMENSIONAL CREATION OF ORIENTAL ANCIENT ART BASED ON BIONICS}

Whatever in the Chinese and Western, the bionic and imitating art has always been one of the most important sorts of Visual Art. In the west, the evolution of Art goes through from original realistic caves mural and bone carvings in the 
Paleolithic period to realism with refining the classical visual languages, then developed to hyperrealism ultimately, the idea behinds it is that representing the natural objects truly. It is an undeniable fact that western art has reached the peak of human art on the way of faithfully reproducing objects, so we used to consider Realistic Art as the main characteristic of western traditional Arts. In fact, the realistic philosophy and skills have been continued from generation to generation and never stopped in China either, for three-dimensional reproduced creation, it mainly shows in kinds of Arts and Crafts works, such as imitating animals jade ware, bionic pottery, bronze ware as well as carving crafts, we can see much of these works and descriptions from relative museums and books. In LvYuanCongHua which was written by QianYong in the Qing Dynasty, there has some description about an excellent sculpture called "ghost craftsman" who was able to engrave figures, tea set, window, even mast of a ship on olive stone and walnut, and everything was manifested as thin as hair but totally lifelike, so the author belauded such proficient skills highly. For two-dimensional art as painting, there also has adequate stating about the idea of realistic expression in generations of painting theories, such as "The most essential factor in judging a painting is realistic, the more lifelike, the better the painting is; otherwise, inferiority." (Song Dynasty, HanQi, ZhiGuiLunHua)

It has been a long tradition in China that the main feature is merging the natural shape into some specific functional parts in making bionic and imitating vessels. There have plenty of ancient examples such as Pottery Eagle Zun in the Yangshao culture ${ }^{1}$, Red Pottery Animal Vessel in the Dawenkou culture ${ }^{2}$, lately animal worships like Bronze Rhinoceros Zun, Ram Zun and Mandarin Ducks Lacquer Dou and so on, all of these are cases which use the internal space of imitation object for storage "Fig. 1", "Fig. 2", "Fig. 3".

1 The Yangshao culture was a Neolithic culture that existed extensively along the Yellow River in China. It is dated from around $5000 \mathrm{BC}$ to 3000 BC. The culture is named after Yangshao, the first excavated representative village of this culture, which was discovered in 1921 in Henan Province by the Swedish archaeologist Johan Gunnar Andersson (1874-1960). The culture flourished mainly in the provinces of Henan, Shaanxi and Shanxi.

2 The Dawenkou culture is a name given by archaeologists to a group of Neolithic communities who lived

primarily in Shandong, but also appeared in Anhui, Henan and Jiangsu, China. The culture existed from 4100 to $2600 \mathrm{BC}$, co-existing with the Yangshao culture. Turquoise, jade and ivory artefacts are commonly found at Dawenkou sites. The earliest examples of alligator drums appear at Dawenkou sites. Neolithic signs, perhaps related to subsequent scripts, such as those of the Shang Dynasty, have been found on Dawenkou pottery.

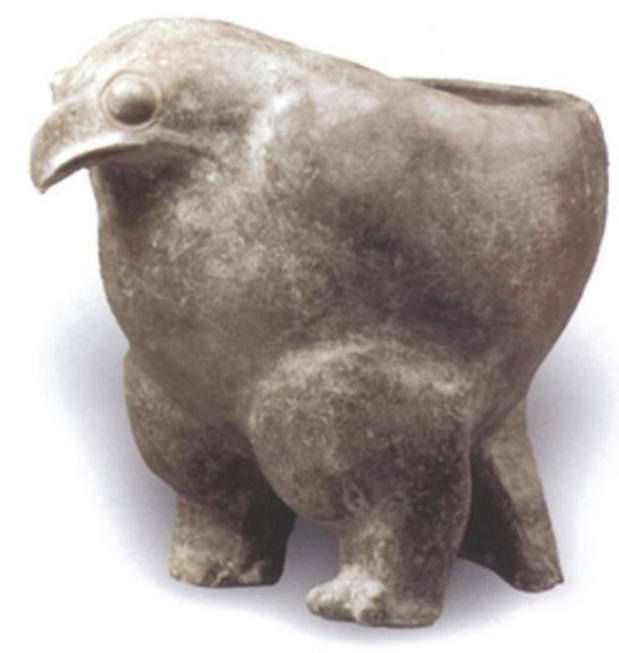

Fig. 1. Pottery Eagle Zun, Yangshao culture

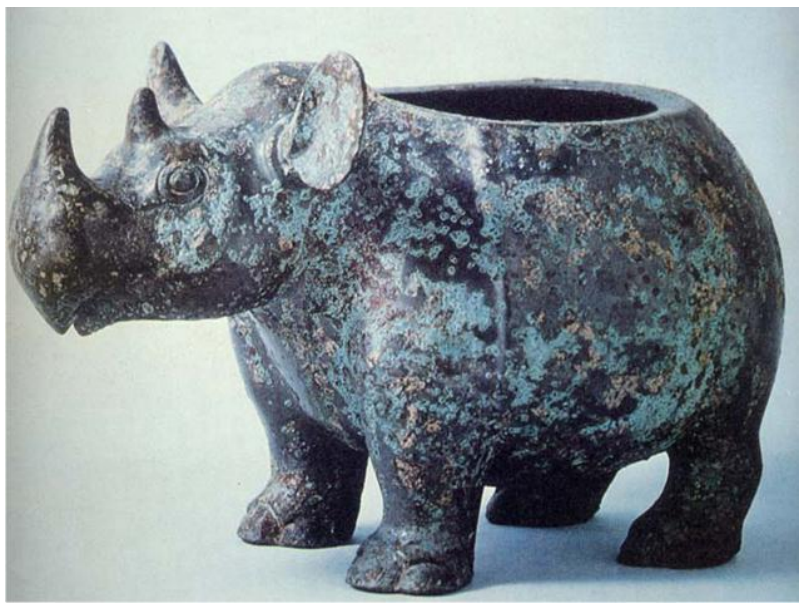

Fig. 2. Bronze Rhinoceros Zun, Shang Dynasty

\section{THE RED STONEWARE ART OF JIANG RONG}

Making a comprehensive survey from ancient to modern times all over the world, we've rarely seen artistic works could perfectly imitated in shape, color and spirit in spite of there have abound of bionic and imitating cases. The reasons behind it are not only some objective factors but also depends on the abilities of manipulating various elements of Plastic Arts. For some objective factors, the plasticity of materials and limitation of craftsmanship are major obstacles, take bronze, gold, silver, jade and stone for instance, these materials can be made in lively shapes but difficult in colors and spirit which based on the former two elements, but red stoneware clay can be molded to achieve realistic effects in shapes, colors and spirit by matching colors skillfully, and this is a natural advantage in creating bionic and imitating vessels with red stoneware clay. As a sort of Chinese Ceramic Art, there has few prominent artists in making "HuaHuo" of YiXing Red Stoneware due to its short history, generally we consider GongChun`s ShuYing Teapot in the 
Ming Dynasty as the earliest example of excellent work, then the most famous artist is Chen Mingyuan in the early Qing Dynasty; and Yang Fengnian in the late Qing Dynasty has obtained certain achievement either; Besides, there still have some outstanding artists of Red Stoneware Art such as Yu Guoliang (1874-1939), Chen Guangming (1859-1930) in the late Qing Dynasty and Pei Shiming (1892-1976) in the contemporary, but the three above have seldom focused on "HuaHuo" and traditional elegance works in general. Until the present time, Jiang Rong`s achievement can be equated with Chen Mingyuan, she devoted herself to create bionic and imitating red stoneware works, built up and expanded on the categories and styles of Chen Mingyuan. In that way, Jiang Rong follows the same craftsmanship but different artistic meaning with Chen Mingyuan, and always maintains her own characteristics. Compared with the two, Chen Mingyuan`s works are of primitive simplicity, spiritual and literary characteristics, and Jiang Rong`s style is full of fresh, native, vivid, brilliant, and expressed within an inch of our lives; Moreover, the women's fine sentiments emitted by her works is unique, beyond the shapes and colors, Jiang Rong`s art give us the psychological experience of similarity in spirit.

The hardest and most important part in realistic art is not achieve a similar appearance but spirit, and Chinese art always pay more attention on the spirit and romantic charm even more. There have sufficient brilliant discussions about this issue from the theoretic level, such as"represent similarity is easy in shape but hard in spirit while painting" (Song Dynasty, YuanWen, WengYou Comments), "an unskillful painter has to cobble together and never gets the similarity in spirit but just parody of some colors, for it lacks of nature interest" (Qing Dynasty, Shen ZongQian, Painting Study of JieZhou) and so on, all of these are views concerning on similarity in spirit. The basis of spiritual similarity is the idea of "all things having souls", generally, it is more understandable that human beings and animals are species with feeling, perception and emotion, so artist may have clear goals to pursue while expressing them, and at the same time audience could realize the artist's talent on spiritual similarity representation. However, some objects such as plants, fruits and vegetables are difficult to be displayed with sense of life. Like a single Chinese chestnut, a single peanut, a section of lotus root, a water chestnut and so on(these are motifs which expressed in Jiang Rong`s red stoneware art frequently), especially without any additional accompaniment (such as vines or leaves), it's a high challenge to represent their vivid energy under this situation. We might even say that the special way of expression is not depends on diligence and skills but genius, and this is the critical difference and barrier between talented artists and familiar craftsmen, moreover, this is the artist's very preciousness. No matter the practical or the traditional elegance works, the most remarkable advantage expressed in Jiang Rong`s bionic red stoneware vessels is not only the proficient craftsmanship but mainly the spiritual similarity, since it's difficult for an ordinary person to achieve "Fig. 3", "Fig. 4", "Fig. 5", "Fig. 6".

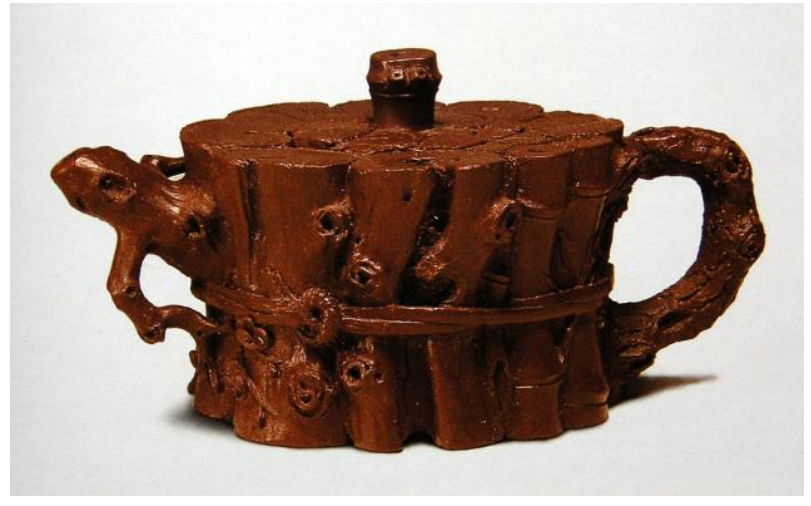

Fig. 3. Purple-clay Pot of Pine-Bamboo-Plum Fardel, Jiang Rong, 1945

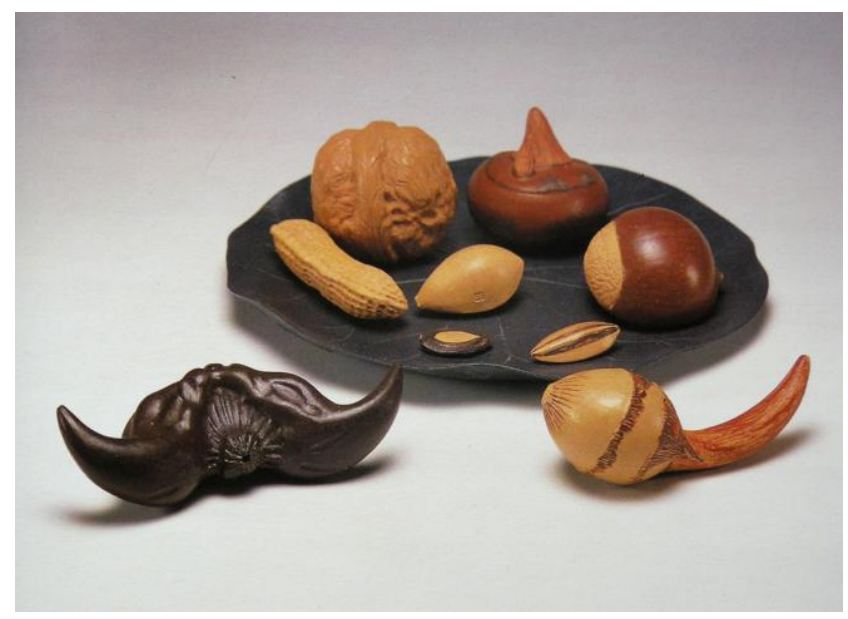

Fig. 4. Nine Pieces Symbolic fruits, Jiang Rong, 1955

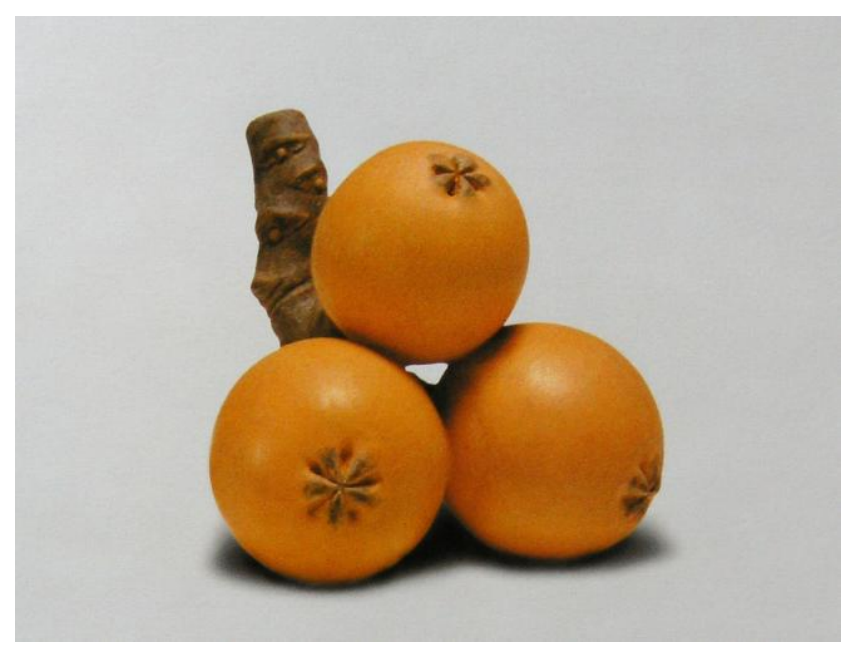

Fig. 5. Teapot Spout with Design of Loquat, Jiang Rong, 1979 


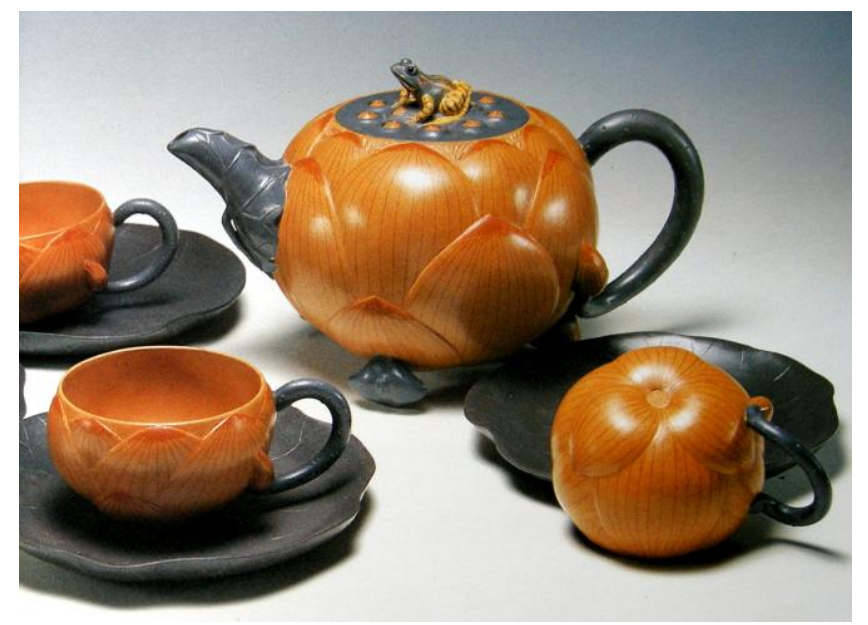

Fig. 6. Nine pieces Tea Set with Design of Water Lily, Jiang Rong, 1955

\section{WHAT`S THE MYSTERY OF EXPRESSING SPIRITUAL SIMILARITY?}

In general, the mystery of artistic creation is hard to be simply revealed, however, there is a critical step of typification in representing the natural objects truly. At first, human beings rely on their instinct to perceive the world around them; then the instinctive experience will be internalized into some paradigms and types gradually; finally, they could generalize diverse objects in real life and draw the quintessence from it by the internalized experience. In fact, the internalized process of realistic experience is typification, everyone own this kind of mechanism but most of them are subconscious and natural towards it. But the artistic typification has to promote the subconscious activities to conscious level, and express it by particular skill and material. For example, it seems to be water chestnut is known for everyone, but after think about it seriously we may realize that no one has seen a "real" and "perfect" water chestnut, for the water chestnuts we have seen in life are different in size and color, that is to say, a true water chestnut in life is not an abstractive but a specific and individual one. The concept has already explained clearly in the early Qin Dynasty by famous school, in artistic creation, a successful typification means to represent the water chestnut through integrating the most notable feature of it. And this is the reason why the red stoneware water chestnut made by Jiang Rong looks like the most perfect one. Actually, Jiang Rong created nine bionic fruit works in this way. The most interesting phenomenon is that every time when we saw a true and beautiful fruit in life, we are admiring:"it is so perfect just as a man-made object!" And this is so called "sculpturesque". But the occurrence of sculpturesque aesthetic experience needs certain works as precondition, which must be created by artists as Jiang Rong.

However, it may not be able to build the spiritual similarity works upon appearance imitating merely, there still needs something mysterious to support it, and the mysterious thing is the manipulation towards the emotional engagement of the object which need to be expressed. Even the most skillful works may reduce to some lifeless specimens without emotional engagement; therefore, the emotional experience is the foundation, which could achieve the spiritual similarity of works. As the archaism saying: "color imitating makes appearance similarity, emotional engagement achieves spiritual similarity" (Eastern Han Dynasty, Wang Yanshou, WenKaoFuHua), which has presented the truth. For artists, all the objects have their respective spirit and soul, so what they need to express in creation is not rigescent appearance but the spirit and soul behind various objects. But as everyone knows, some objects such as plants, fruits and vegetables doesn`t own the spirit and soul physically, so here the "spirit and soul" derives from the artist's emotional engagement because of day after day accompany with them. Moreover, the process that merging the inmost emotion into works depends on extremely sensitive experience. In Jiang Rong`s artistic creation, the expression of spirit is always in a subtle, skillful and natural way. The artistic pursuit of Jiang Rong is not bleak and primitive form which is respected by Chinese traditional literati, but the style full of fun, fresh and gorgeous, and all her works(works of imitating plants, fruits and vegetables) are fresh, plump, bright and mouthwatering things, which seems like just picked from trees and fields right now. Apart from introducing the natural shapes and colors, her creation of bionic teapots also make use of vines and leaves to match up and set off the bodies appropriately. In this way, the teapots` features could be highlighted visibly, besides, these accompaniments can be made into spout, handle and bottom of a teapot perfectly. The sense of life which lies in Jiang Rong`s teapots are always expressed through the elaborate design and retroflex forms, they are indeed lifelike and realistic.

Jiang Rong`s works make us fully experience that the close relationships between Art and $\mathrm{Craft}^{3}$. In Chinese Arts and Crafts field ${ }^{4}$, craftsmanship is widely recognized and even regarded as the most critical valuation criteria, it is understandable. Whereas under the influence of traditional thinking of "Stress on Dao (abstract ideas) More Than Qi (specific skills)" for a long time, craftsmanship was excluded from elegant Arts due to its craft-like, and those Arts and Crafts woks just as elegant and ornamental things for playing and amusing by emperors and literati. Under this situation, Arts and Crafts were never understood as a main part of art history. Although YiXing Red Stoneware Art occupies more prominent position than other craft categories and has certain literature to describe it due to literati`s favor, it still regards

3 Here "Art" and "Craft" are two different terms.

4 Here the "arts and crafts" is a whole concept. It is common knowledge that the arts and crafts are not only the embodiment of the people's longing for aesthetic beauty for themselves and as gifts for others, but also great treasures for China and the rest of the world. Many of the master artisans have had their skills handed down via one generation to the next so that only the offspring of such an artisan could learn the necessary skills required. The result being that they are the most valuable treasures both for a family and for the nation.Of all the Chinese arts and crafts, the most representative are Bronze Vessels, Folk Toys, Embroidery, Calligraphy, Music, Opera, Painting, Cloisonne, Jade, Kites, Lacquer Ware, PaperCuttings, Porcelain, Pottery, Seals, and Silk. They are not only a vivid reflection of the culture of China but also the embodiment of both the local people, and of the nation itself. 
as a kind of skillful crafts, which always held by literati and officials for amusing. In terms of the skills of Arts and Crafts, there have mixed reviews, someone belaud its excellent craftsmanship while others belittle it as something of petty tricks, but we should realize that the ancients`views, no matter praise or blame, the starting point behinds them are not based on essence of art but serving politics or reflecting social and economic situations and so on. Hence, it's necessary to reunderstand Arts and Crafts from Art eyes, only in this way can we acquire its essential value.

\section{CONCLUSION}

In the field of artistic creation, craft and art are two sides of one item, which means they can not be separated into isolated individuals. However, the requirement of craft depends on different needs. For Fine Arts, the craft is directly related to the artist's aesthetic pursuit, which means there has no unitive craftsmanship. On the other hand, for the creation of Arts and Craft, the craft includes two different parts, one is use for meeting special functional requirements, and it has consistent evaluation criterions among the same craft categories; the other is use for achieving special artistic purpose, it depends on artists` intentions, and has different details and criterions. Take Jiang Rong`s work of "Lotus Leaf Teapot" for instance, the craftsmanship for functional requirement is as same as other teapots in different forms, however, there still require distinctive craftsmanship to merge the bionic lotus leaf into the appearance of teapot and display the vitality and energy of it. Only when the artist owns distinguished artistic taste as well as extraordinary vision, can he use the craft to express his ideas perfectly. From this level, Art is actually a special Craft. This understanding is coincide with the meaning of the word "Art", which comes from the field of western art and has widely used by us nowadays. Although the value and status of Arts and Crafts has been belittled due to the neglect of crafts in artistic creation in the past, but Jiang Rong`s achievement has again demonstrated that only the distinctive and excellent craft could match and support artists`idea and philosophy appropriately.

\section{REFERENCES}

[1] Feng Xu, Artificial Identity - The Red Stoneware Artist`s Biography of Jiang Rong, Shanghai Literature \& Art, 2012.

[2] Qian Wu, Records of Prominent Pottery in YangXian, Qing dynasty. 